\title{
INSERÇÃO DOS FONOAUDIÓLOGOS NO SUS/MG E SUA DISTRIBUIÇÃO NO TERRITÓRIO DO ESTADO DE MINAS GERAIS
}

\author{
Insertion of speech therapists in SUS/MG \\ and their distribution in Minas Gerais state
}

\author{
Juliana Nunes Santos ${ }^{(1)}$, Fernanda Jorge Maciel (2), Vanessa de Oliveira Martins ${ }^{(3)}$, \\ Ana Luiza Vilar Rodrigues ${ }^{(4)}$, Ana Flávia Gonzaga ${ }^{(5)}$, Liliane Ferreira da Silva ${ }^{\left({ }^{(6)}\right.}$
}

\section{RESUMO}

Objetivo: investigar a distribuição dos fonoaudiólogos no estado de Minas Gerais, sua inserção no SUS e as variações geográficas dessa distribuição e suas desigualdades. Método: análise dos Cadernos de Informações de Saúde dos 853 municípios do estado de Minas Gerais referentes a 2009, disponíveis no Sistema de Informações em Saúde brasileiro, o DATASUS. Foram pesquisados os indicadores: população municipal, número total de fonoaudiólogos da rede SUS e da rede privada e número médio de fonoaudiólogos (SUS e rede privada) por mil habitantes. Resultados: a análise dos dados revelou a presença de 1.733 fonoaudiólogos atuando no estado em 2009. Destes, 67,8\% atendiam à rede SUS. Dos 853 municípios, 505 (59\%) não possuíam o profissional fonoaudiólogo no período investigado. Observou-se que entre as 13 macrorregiões estaduais as regiões CentroSul e Sul apresentaram a melhor média de fonoaudiólogos por 10.000 habitantes $(1 / 10.000)$ e as regiões Norte de Minas e Nordeste, as piores: 0,16 e 0,05/10.000, respectivamente. Observou-se a presença de 0,58 fonoaudiólogos/10.000 habitantes disponíveis na rede SUS e 0,86 fonoaudiólogos/10.000 atendendo à rede privada e ao SUS no estado. Conclusão: a inclusão de fonoaudiólogos na assistência à saúde estadual ainda é deficitária, sendo observada grande disparidade na distribuição dos profissionais. É notório o estrangulamento da assistência fonoaudiológica no SUS em Minas Gerais, visto que para cada 17.000 mineiros existia somente um fonoaudiólogo no SUS estadual em 2009. Ressalta-se a necessidade de uma mobilização dos profissionais e dos gestores de saúde para garantir a integralidade da atenção à saúde no estado.

DESCRITORES: Fonoaudiologia; SUS; Atenção à Saúde; Acesso aos Serviços de Saúde

(1) Fonoaudióloga; Professora Adjunto do curso de Fonoaudiologia da Universidade Federal de Minas Gerais - UFMG, Minas Gerais, Brasil; Doutora em Ciências da Saúde pela Universidade Federal de Minas Gerais.

(2) Fonoaudióloga da Secretaria de Estado de Saúde de Minas Gerais, Brasil; Mestranda em Saúde Coletiva pela Universidade Federal de Minas Gerais, Minas Gerais, Brasil.

(3) Fonoaudióloga; Professora Adjunto do curso de Fonoaudiologia da Universidade Federal de Minas Gerais UFMG, Minas Gerais, Brasil; Doutora em Linguística pela Universidade de São Paulo.

(4) Acadêmica do curso de Fonoaudiologia da Universidade Federal de Minas Gerais - UFMG, Minas Gerais, Brasil.

(5) Acadêmica do curso de Fonoaudiologia da Universidade Federal de Minas Gerais - UFMG, Minas Gerais, Brasil.

(6) Acadêmica do curso de Fonoaudiologia da Universidade Federal de Minas Gerais - UFMG, Minas Gerais, Brasil.

Conflito de interesses: inexistente

\section{INTRODUÇÃO}

A criação de um sistema de seguridade social no Brasil se deu com a promulgação da Constituição de 1988. O Sistema Único de Saúde (SUS) teve, por sua vez, sua regulamentação institucionalizada a partir da publicação das leis 8080/90 e 8142/90, que definem seus três princípios doutrinários: universalidade, equidade e integralidade ${ }^{1}$.

A Fonoaudiologia - profissão à qual compete a promoção da saúde, prevenção e tratamento de agravos referentes a aspectos fonoaudiológi$\cos ^{2}$ - está gradativamente ocupando espaço nos serviços públicos de saúde, principalmente a partir da década de 1980, por intermédio das Secretarias 
de Educação e Saúde. Nessa época, no entanto, existiam poucos profissionais da área e esses apresentavam uma visão clínica, decorrente da própria formação acadêmica, com foco na reabilitação ${ }^{3}$. Essa forma de atuação comprometeu a efetividade do serviço, pois não eram realizadas propostas de atuação para um grande número de pessoas, ficando o fonoaudiólogo concentrado em ambulatórios de saúde mental e hospitais ${ }^{3-5}$.

Com a criação do SUS, os fonoaudiólogos tiveram a oportunidade de expandir sua atuação, até então focada nas práticas reabilitadoras, para os diversos níveis de assistência à saúde, incluindo a atenção básica ${ }^{4,6}$. Desde então, observam-se fonoaudiólogos atuando em todos os níveis de atenção com ações de promoção, proteção e recuperação da saúde nos diversos aspectos relacionados à comunicação humana. Sua inserção é observada em Unidades Básicas de Saúde, Ambulatórios de Especialidades, Hospitais, Unidades Educacionais, domicílios e outros recursos da comunidade ${ }^{3,5,7}$, conseqüência do reconhecimento da relevância dos distúrbios de comunicação, já que estes podem comprometer o desenvolvimento infantil, restringir a realização profissional e prejudicar o bem-estar econômico da sociedade ${ }^{6-8}$. Além disso, a inserção do fonoaudiólogo neste novo contexto despertou nele o interesse por pesquisas sobre as desordens da comunicação na população ${ }^{9}$, pois existem o desejo e a necessidade de formalizar sua inserção nos demais serviços do SUS. As atuais reformas na graduação evidenciam a necessidade de mudar a formação deste profissional, a fim de torná-lo apto a atuar em saúde pública ${ }^{10}$.

Nos últimos anos, políticas nacionais de saúde favoreceram a inserção do fonoaudiólogo no SUS, especialmente na atenção básica. A criação dos Núcleos de Apoio em Saúde da Família (NASF) retrata e assegura essa inserção, levando o fonoaudiólogo a embasar sua atuação numa prática intersetorial e interdisciplinar, considerando o indivíduo e sua inserção na família e na comunidade ${ }^{11}$. Atualmente, observam-se fonoaudiólogos inseridos em diferentes ações e programas do SUS, atuando com ações na estratégia de Saúde da Família, saúde infantil, saúde mental, saúde escolar e do adolescente, e com especial atuação na Política Nacional de Saúde Auditiva 3,12-14. Diante das mudanças, muitos conceitos e práticas têm sido reavaliados, a partir da intenção de oferecer um serviço de qualidade e em conformidade com os preceitos da saúde pública. Para isso, é preciso apresentar evidências científicas sobre a importância deste trabalho e das transformações decorrentes destas intervenções ${ }^{15}$. A preocupação com a formação do fonoaudiólogo, com a conduta clínica ${ }^{12,16}$ e com a distribuição dos profissionais nos serviços públicos e privados, visando a uma maior cobertura das demandas populacionais, também está presente em outros países, como na África do Sul ${ }^{16,17}$ e no restante do mundo 9 .

Nesse sentido, a avaliação de políticas e programas é essencial em saúde pública, contribuindo para os esforços em busca de uma sociedade mais saudável e prevenindo o desperdício de recursos com a implantação de programas ineficazes. Medidas epidemiológicas frequentemente são utilizadas como subsídios à formulação e avaliação das políticas públicas, podendo ser utilizadas como ferramentas de apoio ao planejamento em saúde nas esferas municipal, estadual e federal ${ }^{8,18-20}$.

No âmbito da gestão estadual, de modo a garantir o acesso da população aos serviços de saúde, são consideradas diversas variáveis no processo de organização dos níveis de complexidade tecnológica dos serviços do SUS.

O estado de Minas Gerais, que possui população de aproximadamente 20 milhões de habitantes e 853 municípios, tem seu território organizado em 75 microrregiões e 13 macrorregiões de saúde, conforme Plano Diretor de Regionalização vigente no ano de $2008^{21,22}$. O planejamento em saúde em Minas Gerais busca organizar os serviços na lógica das redes de atenção, tendo a atenção primária como coordenadora do processo de cuidado em relação aos demais pontos de atenção do sistema ${ }^{23}$. Os objetivos prioritários na atual gestão do SUS em Minas são reduzir a mortalidade infantil, melhorar a capacidade funcional dos idosos e reduzir as mortes por causas externas, doenças do aparelho circulatório e diabetes ${ }^{21}$. Além disso, a gestão estadual em Minas coordena a implantação da Rede de Atenção à Saúde Auditiva, também organizada conforme o Plano Diretor de Regionalização, em que o profissional fonoaudiólogo atua de forma descentralizada para a melhoria da qualidade de vida da população com deficiência auditiva e outros distúrbios da comunicação ${ }^{21}$.

Para facilitar a quantificação e a avaliação da situação sanitária, foram desenvolvidos indicadores de saúde, os quais são medidas-síntese que contêm informação relevante sobre determinados atributos e dimensões do estado de saúde, bem como do desempenho do sistema de saúde. O processo de formulação de indicadores de saúde não é simples, envolvendo métodos epidemiológicos rigorosos. Nos últimos anos, a Fonoaudiologia vem apontando seus próprios indicadores, relacionados a: precisão diagnóstica, tempo de terapia e qualidade dos métodos de tratamento. Entretanto, indicadores para a avaliação institucional na área não foram 
encontrados. Além disso, a distribuição dos fonoaudiólogos é bastante desigual no País, sendo este um importante indicador de atendimento aos distúrbios da comunicação. Tais informações são fundamentais, associadas às demandas populacionais para a alocação de recursos para a contratação de pessoal $9,20,24,25$.

Diante da carência de estudos sobre a inserção do fonoaudiólogo na rede de saúde no país, pretende-se, neste artigo, investigar a distribuição dos fonoaudiólogos no estado de Minas Gerais, sua inserção no SUS, assim como analisar as variações geográficas dessa distribuição e suas desigualdades.

\section{MÉTODO}

Trata-se de um estudo transversal, realizado no período de abril a junho de 2010. A coleta de dados foi feita por meio da análise dos Cadernos de Informações de Saúde (versão de fevereiro de 2009) dos 853 municípios de Minas Gerais, referentes ao ano de 2009, disponíveis no Sistema de Informações em Saúde brasileiro, o DATASUS ${ }^{26}$. Os Cadernos são atualizados periodicamente e consistem em uma planilha referente a todos os municípios e estados brasileiros, contendo indicadores das diversas bases de dados do Ministério da Saúde. Porém, eventualmente, as bases de dados dos municípios e estados podem estar mais atualizadas, gerando diferenças entre os indicadores disponibilizados em relação aos dados do site DATASUS e aos dados calculados pelos estados e municípios a partir de suas bases de dados. Na época da coleta de dados, as informações referentes ao ano de 2009 eram as mais atuais disponíveis no site DATASUS.

Para a análise dos Cadernos de Informações de Saúde consideraram-se os seguintes indicadores: população do município, número total de fonoaudiólogos da rede SUS e da rede privada e número médio de fonoaudiólogos (SUS e rede privada) por mil habitantes.

Foi realizada a análise da distribuição dos profissionais de saúde considerando os territórios de saúde de Minas Gerais (macro e microrregiões), uma vez que existem significativas diferenças regionais referentes a aspectos socio-econômicos e demográficos que podem influenciar também a oferta de serviços de saúde à população e os recursos humanos disponíveis.

O presente estudo foi aprovado pelo Comitê de Ética em Pesquisa da Universidade Federal de Minas Gerais conforme o parecer de no. 0483.0.203.000-10.

Após a coleta de dados, criou-se um banco de dados específico para este estudo no Microsoft Office Excel 2003. Utilizou-se o teste Qui-quadrado para verificar as diferenças entre as proporções, com nível de significância de $p<0,05$.

\section{RESULTADOS}

A análise dos dados revelou a presença de 1.733 fonoaudiólogos atuando no estado de MG em fevereiro de 2009. Destes, $67,8 \%$ atendiam à rede SUS. Dos 853 municípios mineiros, 505 (59\%) não possuíam o profissional fonoaudiólogo no período investigado.

Observou-se que entre as 13 macrorregiões do estado, a Centro-Sul e a Sul apresentaram a melhor média de fonoaudiólogos por 10.000 habitantes (1/10.000) e as regiões Norte de Minas e Nordeste, as piores, 0,16 e 0,05/10.000, respectivamente (Tabela 1).

Nos municípios com serviço de fonoaudiologia, a média de profissionais foi de 4,9 por município, sendo que 240 municípios possuem entre um ou dois fonoaudiólogos na assistência à saúde da população do município (Tabela 2). Os municípios "pólos" das macrorregionais apresentaram o maior número de profissionais fonoaudiólogos na atenção à saúde (Tabela 3).

A relação entre a presença do fonoaudiólogo na assistência à saúde do município e o número de habitantes da cidade pode ser visualizada na Tabela 4.

A distribuição dos fonoaudiólogos segundo as microrregiões de saúde no estado pode ser visualizada na Figura 1. 
Tabela 1 - Distribuição dos fonoaudiólogos no estado de Minas Gerais no ano de 2009, segundo as macrorregionais de saúde

\begin{tabular}{lcccccc}
\hline Macrorregional & $\begin{array}{c}\text { Total da } \\
\text { população Fonoaudiólogos }\end{array}$ & $\begin{array}{c}\text { Total de } \\
\text { Fonoaudiólogos } \\
\text { por } \mathbf{1 0 . 0 0 0 / h a b}\end{array}$ & $\begin{array}{c}\text { Média de } \\
\text { Fonoaudiólogos/SUS }\end{array}$ & $\begin{array}{c}\text { Média de } \\
\text { Tonoaudiólogos/ } \\
\text { SUS por } \\
\mathbf{1 0 . 0 0 0 / h a b}\end{array}$ \\
\hline Sul & 2.609 .586 & 337 & 1.00 & 240 & $71,2 \%$ & 0,92 \\
Centro-Sul & 755.761 & 122 & 1.00 & 99 & $81,1 \%$ & 1,31 \\
Centro & 6.350 .883 & 580 & 0.62 & 327 & $56,4 \%$ & 0,51 \\
Jequitinhonha & 280.338 & 14 & 0.24 & 14 & $100,0 \%$ & 0,50 \\
Oeste & 1.178 .150 & 98 & 0.64 & 62 & $63,3 \%$ & 0,53 \\
Leste & 1.469 .504 & 75 & 0.19 & 38 & $50,7 \%$ & 0,26 \\
Sudeste & 1.589 .255 & 149 & 0.74 & 106 & $71,1 \%$ & 0,67 \\
Norte de Minas & 1.609 .862 & 87 & 0.16 & 60 & $69,0 \%$ & 0,37 \\
Noroeste & 657.489 & 43 & 0.38 & 39 & $90,7 \%$ & 0,59 \\
Leste do Sul & 666.733 & 37 & 0.52 & 34 & $91,9 \%$ & 0,51 \\
Nordeste & 917.626 & 19 & 0.05 & 19 & $100,0 \%$ & 0,21 \\
Triângulo do Sul & 683.061 & 83 & 0.86 & 70 & $84,3 \%$ & 1,02 \\
Triângulo do Norte & 1.231 .518 & 89 & 0.94 & 65 & $73,0 \%$ & 0,53 \\
Minas Gerais & 19.999 .766 & 1.733 & 0,86 & 1.173 & $67,7 \%$ & 0,59 \\
\hline
\end{tabular}

Fonte: Cadernos de Informações de saúde, versão de fevereiro de 2009. DATASUS, Ministério da Saúde.

Tabela 2 - Distribuição dos municípios onforme o número de fonoaudiólogos no estado de Minas Gerais no ano de 2009

\begin{tabular}{cc}
\hline Número de fonoaudiólogos & Quantitativo de municípios \\
\hline 1 & 190 \\
2 & 50 \\
$>2 \mathrm{e} \leq 5$ & 57 \\
$>5 \mathrm{e} \leq 10$ & 22 \\
$>10$ & 29 \\
\hline 1 & 348 \\
\hline
\end{tabular}

Fonte: Cadernos de Informações de saúde, versão de fevereiro de 2009. DATASUS, Ministério da Saúde. 
Tabela 3 - Análise da distribuição dos fonoaudiólogos nas cidades pólo das macrorregiões de saúde do estado de Minas Gerais no ano de 2009

\begin{tabular}{lccc}
\hline Pólos das Macrorregiões & Total população & $\begin{array}{c}\text { Total de } \\
\text { Fonoaudiólogos }\end{array}$ & $\begin{array}{c}\text { Média } \\
\text { Fonoaudiólogos } \\
\text { por 10.000/hab }\end{array}$ \\
\hline Montes Claros - Norte de Minas & 363.226 & 58 & 1,60 \\
Téofilo Otoni - Nordeste & 130.519 & 12 & 0,92 \\
Governador Valadares - Leste do Norte & 263.278 & 25 & 0,95 \\
Ipatinga - Leste do Norte & 244.509 & 23 & 0,94 \\
Ponte Nova - Leste do Sul & 57.657 & 9 & 1,56 \\
Juiz de Fora - Sudeste & 526.709 & 61 & 1,16 \\
Passos - Sul & 107.617 & 20 & 1,86 \\
Varginha - Sul & 121.788 & 13 & 1,07 \\
Alfenas - Sul & 75.213 & 15 & 1,99 \\
Poços de Caldas - Sul & 151.454 & 43 & 2,84 \\
Pouso Alegre - Sul & 127.075 & 24 & 1,89 \\
Uberaba - Triângulo do Sul & 296.259 & 42 & 1,42 \\
Uberlândia - Triângulo do Norte & 634.349 & 42 & 0,66 \\
Patos de Minas - Noroeste & 139.840 & 12 & 0,86 \\
Divinópolis - Oeste & 216.100 & 16 & 0,74 \\
Barbacena - Centro-sul & 128.572 & 40 & 3,11 \\
Diamantina - Jequitinhonha & 46.374 & 8 & 1,73 \\
Belo Horizonte - Centro & 2.452 .612 & 358 & 1,46 \\
\hline
\end{tabular}

Fonte: Cadernos de Informações de saúde, versão de fevereiro de 2009. DATASUS, Ministério da Saúde.

Tabela 4 - Análise da distribuição dos fonoaudiólogos no estado de Minas Gerais no ano de 2009 segundo tamanho populacional

\begin{tabular}{|c|c|c|c|c|c|c|c|}
\hline & & \multicolumn{2}{|c|}{$\begin{array}{l}\text { Cidades mineiras com } \\
\text { população }\end{array}$} & \multirow[b]{2}{*}{$\begin{array}{l}\text { Razão de } \\
\text { Chances }\end{array}$} & \multirow{2}{*}{$\begin{array}{l}\text { Intervalo } \\
\text { de } 95 \% \text { de } \\
\text { confiança }\end{array}$} & \multirow[b]{2}{*}{$\begin{array}{l}\text { Teste Qui- } \\
\text { Quadrado }\end{array}$} & \multirow[b]{2}{*}{ Valor de $p$} \\
\hline & & $\begin{array}{c}<10.000 \\
\text { habitantes } \\
(n=490)\end{array}$ & $\begin{array}{c}>10.000 \\
\text { habitantes } \\
(n=363)\end{array}$ & & & & \\
\hline \multirow{2}{*}{ Fonoaudiólogo } & $\operatorname{sim}$ & 114 & 233 & \multirow{2}{*}{5.91} & \multirow{2}{*}{$4.33-8.07$} & \multirow{2}{*}{143.01} & \multirow{2}{*}{$0,000^{*}$} \\
\hline & não & 376 & 130 & & & & \\
\hline \multirow{2}{*}{$\begin{array}{l}\text { Fonoaudiólogo } \\
\text { na rede SUS }\end{array}$} & $\operatorname{sim}$ & 109 & 227 & \multirow{2}{*}{5.83} & \multirow{2}{*}{$4.27-7.98$} & \multirow{2}{*}{140.09} & \multirow{2}{*}{$0,000^{*}$} \\
\hline & não & 381 & 136 & & & & \\
\hline
\end{tabular}

Fonte: Cadernos de Informações de saúde, versão de fevereiro de 2009. DATA SUS, Ministério da Saúde. 


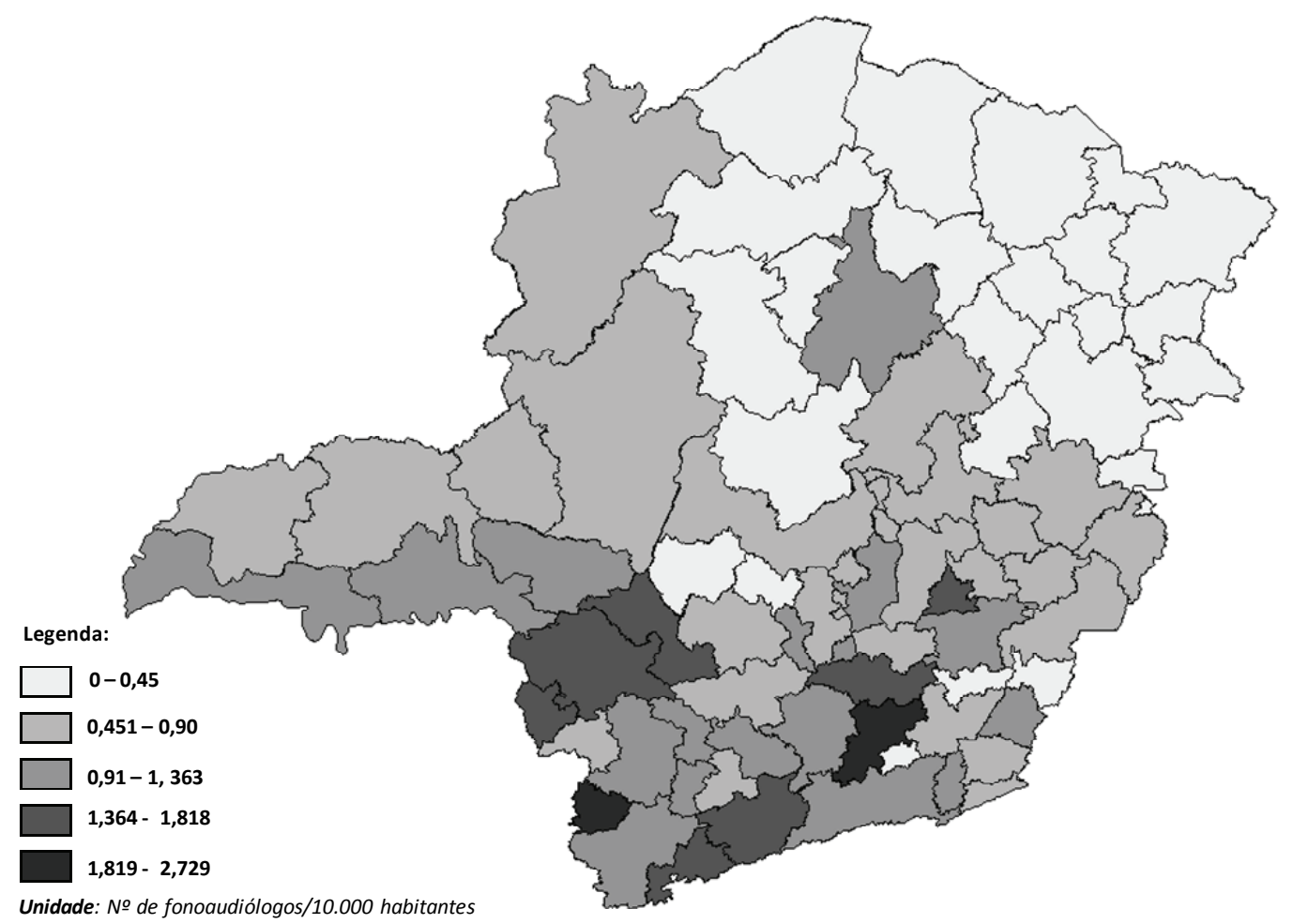

Fonte: Ministério da Saúde/DataSUS - cadernos de informações de saúde, versão fevereiro de 2009. Cadastro Nacional dos Estabelecimentos de Saúde do Brasil - CNES. Ocupações segundo CBO 2002 - Ocupações de Nível Superior: 223810 - Fonoaudiólogo.

Figura 1 - Distribuição de fonoaudiólogos por mil habitantes nas microrregiões de saúde do estado de minas gerais, 2009

\section{DISCUSSÃO}

Este estudo teve por objetivo investigar a distribuição dos fonoaudiólogos no estado de Minas Gerais, levando-se em conta sua inserção no SUS, as variações geográficas dessa distribuição e suas desigualdades, a partir da análise dos Cadernos de Informações de Saúde dos 853 municípios de Minas Gerais. Tais informações estão disponíveis no Sistema de Informações em Saúde brasileiro, o DATASUS.

No Brasil, os sistemas de informação em saúde estão em constante crescimento, apresentando dados de saúde resultantes de inquéritos domiciliares de saúde, planilhas de atendimento nos estabelecimentos de saúde e cadastros de profissionais e de estabelecimentos, dentre outros. Tais informações são fundamentais, mas insuficientes para responder às necessidades da gestão ${ }^{19}$. $\mathrm{Na}$ Fonoaudiologia, tais informações são escassas e as disponíveis são pouco utilizadas.

De acordo com dados disponibilizados pelo CRFa 6 ${ }^{\underline{a}}$ região por consulta eletrônica em março de 2010, o número de fonoaudiólogos inscritos e que atuavam no estado de Minas Gerais até 31 de janeiro de 2009 era de 3.173 profissionais. Isso mostra a fragilidade dos sistemas de informação disponibilizados pelo governo, bem como as limitações dos nossos serviços. De maneira geral, os fonoaudiólogos cadastrados no Cadastro Nacional de Estabelecimentos de Saúde (CNES) compreendem aqueles que atuam em estabelecimentos de saúde. As empresas públicas têm uma maior preocupação com este dado, devido ao faturamento do SUS. Esta acaba não sendo a realidade dos fonoaudiólogos que atuam apenas em consultórios particulares. Muitos desconhecem tal sistema de informação, bem como a sua importância para as estatísticas de saúde. Refazendo os cálculos da distribuição dos fonoaudiólogos, tem-se que 37\% dos fonoaudiólogos de Minas Gerais atuam no SUS, enquanto que, de acordo com os dados disponíveis nos Cadernos de Informações de Saúde de 2009, $67 \%$ atuam no SUS.

Como exposto nos resultados (Tabela 1), este estudo encontrou a maior taxa de fonoaudiólogos nas macrorregionais Sul e Centro-sul (1.0 Fono/10.000hab). Estas macrorregionais possuem, respectivamente, a terceira e a sexta maiores médias de PIB per capta do estado ( $\mathrm{R} \$ 9317,92$ 
e $\mathrm{R} \$ 9317,92)$, além de deterem altos índices de alfabetização (79,76\% e 79,76\%). A macrorregional Nordeste, que possui a menor porcentagem de fonoaudiólogos (0.05 Fono/10.000hab), é também a com maior população sem acesso à instalação sanitária $(24,03 \%)$ e menor média do PIB per capita do estado $(R \$ 3823,20)$. Macrorregionais de alto desenvolvimento, com altas taxas de alfabetização e as maiores médias do PIB per capita, como se vê no caso do Triângulo do sul e do Triângulo do norte, possuem também uma alta média de fonoaudiólogos (0.86 e 0.94 Fono/10.000hab) em detrimento das outras macros ${ }^{26}$.

No Plano Diretor de Regionalização de Minas Gerais (PDR/MG), a cada Nível de Atenção corresponde preponderantemente um Território Sanitário: ao município compete a Atenção Primária e não apenas a "Atenção Básica"; à Atenção Secundária, o Nível Microrregional, com ocorrência da maioria dos procedimentos no município polo micro; à Terciária, o Nível Macrorregional, com os serviços concentrados na cidade pólo ${ }^{27}$. Dessa forma, acredita-se que o maior número médio por 10 mil habitantes de fonoaudiólogos nos polos macrorregionais (Tabela 3) deve-se ao papel que tais municípios exercem na organização regionalizada do sistema de saúde no estado.

Além disso, com a criação dos Núcleos de Apoio à Saúde da Família (NASF) ${ }^{11}$, que inclui o fonoaudiólogo nessa equipe, observa-se também uma maior concentração dessas equipes nas cidades polo. Atualmente, 9 dos 18 municípios polo possuem pelo menos duas equipes de NASF, de acordo com informações cedidas pela Gerência de Atenção Primária da Secretaria de Estado de Saúde de Minas Gerais em maio de 2010.

Ao relacionar a presença do profissional fonoaudiólogo na atenção à saúde, independentemente de o mesmo estar ou não vinculado ao SUS, com o número de habitantes do município, observouse uma relação com significância estatística $(p<$ 0,001 ), mostrando a concentração do profissional em cidades com maior concentração populacional (Tabela 4). Possível explicação para o fato precede da própria estrutura organizacional dos serviços do SUS em níveis de complexidade. No entanto, com base em tais considerações, pode-se dizer que os princípios do SUS - equidade, universalidade e integralidade - estão sendo infringidos ${ }^{28}$. Como bem discutido por Moreira e Mota $^{3}$, a comunicação, sendo objeto de estudo da Fonoaudiologia, merece importante atenção das ações de saúde pública, uma vez que possibilita ao indivíduo se colocar como agente transformador da sociedade e de sua realidade.
Neste estudo, observou-se grande disparidade na distribuição do fonoaudiólogo no estado de Minas Gerais (Figura 1). O termo desigualdades, em saúde, abrange as diferenças, as variações e as disparidades nos atendimentos em saúde das pessoas e grupos ${ }^{29}$, sendo um problema encontrado em todos os países que se propuseram a medi-la ${ }^{30,31}$.

De maneira geral, o número de fonoaudiólogos no estado de Minas Gerais está aquém do necessário. Lessa e Miranda apontam que, para garantir a atenção universal, integral e equânime, um critério proposto para estimar a necessidade de profissionais seria 1 fonoaudiólogo para 10.000 habitantes na atenção básica, 1 para 50.000 habitantes para a atenção especializada de média complexidade e 1 para 100.000 habitantes para alta complexidade ${ }^{32}$. Fazendo uma estimativa do número necessário de fonoaudiólogos no serviço público de Minas Gerais, encontram-se os seguintes valores: 2.000 para a atenção básica, 400 para a média complexidade e 200 para a alta complexidade, totalizando 2.600 fonoaudiólogos. Entretanto, o que pode ser observado (Tabela 3) é que apenas as macrorregiões Centro-Sul e Sul apresentam um fonoaudiólogo para cada 10.000 habitantes. Vale considerar que os dados encontrados no DATASUS não se referem apenas aos fonoaudiólogos que atuam na Atenção Básica, o que agrava ainda mais a situação. A falta de fonoaudiólogos no serviço público de saúde pode ser consequência da reforma do Estado brasileiro, em que ocorre um enxugamento (também) do setor saúde, priorizando o foco nas doenças que podem levar a óbito ${ }^{33}$. Portanto, a fonoaudiologia raramente é convocada para trabalhar nos serviços públicos, em virtude da restrição de recursos financeiros, fato que pode colocar a profissão à margem dos reais problemas sanitários enfrentados pela sociedade. A partir disso, a autora considera que a fonoaudiologia favorece as classes que possuem o poder de compra ${ }^{33}$.Buarque e colaboradores ${ }^{34}$ estu- $^{-}$ daram a distribuição de fonoaudiólogos nas macrorregiões brasileiras. Com base na proposta de Lessa e Miranda ${ }^{32}$, sobre a necessidade de um fonoaudiólogo na atenção básica a cada 10.000 habitantes, os autores calcularam a porcentagem de fonoaudiólogos que atuam no SUS em cada macrorregião em relação ao número necessário. De acordo com os resultados, a região Norte apresenta $39,11 \%$ dos profissionais necessários; a Nordeste, 49,35\%,;a Sul, 98,50\%; a Centro-oeste, 83,21\%; e a Sudeste, $110,23 \%{ }^{34}$. Os autores não deixam claro se isso se refere a todos os fonoaudiólogos que atuam no SUS ou apenas àqueles que atuam na atenção básica. Independente disso, os dados encontrados neste estudo mostram que os dados encontrados 
por Buarque e colaboradores não são a realidade da região Sudeste. Provavelmente, os estados de São Paulo e Rio de Janeiro apresentam maior concentração de fonoaudiólogos no SUS, por apresentarem mais profissionais da área.

Este estudo analisou a distribuição do fonoaudiólogo no estado de Minas Gerais a partir da leitura do DATASUS. A vantagem de analisar informações coletadas por meio de dados secundários está na facilidade de realização da pesquisa, uma vez que o acesso a elas torna-se mais ágil, pela sua disponibilidade e, portanto, menos dispendioso em relação a custos e ao tempo para obtê-las ${ }^{35}$. Entretanto, os dados podem estar obsoletos, as informações podem não ser confiáveis e podem existir campos não preenchidos, o que interfere na análise geral do estudo. Em seu estudo para verificar o número de fonoaudiólogos que atuavam no SUS em Salvador no ano de 2006, a autora entrou em contato com as instituições para checar a exatidão das informações. De acordo com os resultados encontrados, apenas em $46,5 \%$ das instituições realmente havia um fonoaudiólogo trabalhando ${ }^{35}$.

Dessa forma, a utilização de sistemas de informação enquanto instrumento de definição do perfil epidemiológico, ações de planejamento e avaliação de serviço passa, primeiramente, pela avaliação da qualidade dos dados e informações geradas ${ }^{36}$. Não resta dúvida de que, frequentemente, os gestores são incapazes de ver através da névoa provocada pelas variáveis de confusão, malclassificadas ou ausentes, o que reforça a necessidade de investir na criação e análise de indicadores de saúde ${ }^{9,24}$, assim como em inquéritos populacionais ${ }^{19}$ e investigações do conhecimento dos usuários sobre o acesso aos serviços de saúde ${ }^{25}$.
Ao ser questionado a respeito de mais contratações, o ministro da Saúde explicou que para contratar mais fonoaudiólogos cabe ao gestor estadual e municipal avaliar a necessidade de ampliar o número de profissionais na rede pública local, considerando os critérios populacionais, epidemiológicos e de serviços ${ }^{37}$. Para isso, são necessários dados epidemiológicos que caracterizem a distribuição das doenças, sistemas de informação efetivos e caracterização da demanda dos serviços de saúde já existentes. Mais pesquisas na área devem ser feitas, especialmente na Fonoaudiologia, a fim de auxiliar gestores e profissionais de saúde na avaliação e implementação de programas e políticas públicas que possam impactar positivamente a saúde da população ${ }^{9}$.

\section{CONCLUSÃO}

A inclusão de fonoaudiólogos na assistência à saúde no estado de Minas Gerais ainda é deficitária, visto que somente $41 \%$ dos municípios dispõem de tal profissional. Observou-se grande disparidade na distribuição dos profissionais, com maior concentração nas cidades polo e nos municípios com mais de 40.000 habitantes. É notório o estrangulamento da assistência fonoaudiológica no SUS no estado, visto que para cada 17.000 mineiros existia somente 1 fonoaudiólogo no Sistema Único de Saúde estadual no ano de 2009. Ressalta-se a necessidade de uma mobilização da classe profissional e dos gestores de saúde para garantir a integralidade da atenção à saúde no estado, em consonância com os princípios doutrinários do SUS, garantidos pela Constituição Federal. 


\begin{abstract}
Purpose: to investigate the distribution of speech therapists in the state of Minas Gerais, their inclusion in SUS (Unique Health System) and analyze the geographical variations of this distribution and its inequalities. Method: analyzing Reports in Health Information of 853 municipalities in Minas Gerais, in the year of 2009, which are available at the Health Information System in Brazil, called DATASUS. The indicators: municipal population, total number of speech therapists in SUS and in the private network and the average number of speech therapists (SUS and private network) per thousand inhabitants were examined. Results: data analysis revealed the presence of 1733 speech therapists acting in the state in 2009. Of these, $67.8 \%$ attended the SUS system. Of the 853 municipalities, 505 (59\%) lacked the professional speech therapist in the investigated period. It was observed that among the 13 state geographical regions, the Centre-South and South regions had the best average of speech therapists per 10,000 inhabitants (1/10.000) and the North of Minas Gerais and Northeast regions, the worst, 0.16 and $0.05 / 10,000$, respectively. IWe observed the presence of 0.58 speech therapists $/ 10.000$ inhabitants available in SUS and 0.86 speech pathologist/10.000 in the private network and health care system in Brazil. Conclusion: the inclusion of speech therapists in the state health care is poor, being observed a great disparity in the distribution of professionals. The bottleneck in the speech therapy care in SUS in Minas Gerais is notorious, since that for every 17000 Minas residents there was only one speech therapist in SUS in the state in 2009. It is important to highlight the need for mobilization of professionals and health managers to ensure health care integrity in the state.
\end{abstract}

KEYWORDS: Speech, Language and Hearing Sciences; Single Health System; Health Care (Public Health); Health Services Accessibility

\section{REFERÊNCIAS}

1. Brasil. Conselho Nacional de Secretários de Saúde. SUS 20 anos./ Conselho Nacional de Secretários de Saúde. Brasília: CONASS, 2009.

2. Conselho Federal de Fonoaudiologia CFFa. Áreas de competência do Fonoaudiólogo no Brasil. Brasília, 2007.

3. Moreira MD, Mota HB. Os caminhos da fonoaudiologia no Sistema Único de Saúde - SUS. Rev. CEFAC. 2009;11(3): 516-21.

4. Befi D. A inserção da fonoaudiologia na atenção primária à saúde. In: Befi $\mathrm{D}$, organizador. Fonoaudiologia na atenção primária à saúde. São Paulo: Lovise; 1997. p.15-36.

5. Lipay MS, Almeida EC. A fonoaudiologia e sua inserção na saúde pública. Rev Ciênc Med 2007;16(1):31-41.

6. Peranich L, Reynolds KB, O'Brien S, Bosch J, Cranfill T. The Roles of Occupational Therapy, Physical Therapy, and Speech/Language Pathology in Primary Care. The Journal for Nurse PractitionersJNP 2010;6(1):26-43.

7. Somefun OA, Lesi FE, Danfulani MA, Olusanya BO. Communication disorders in Nigerian children. Int J Pediatr Otorhinolaryngol. 2006;70(4):697-702.

8. Olusanya BO, Newton VE. Global burden of childhood hearing impairment and disease control priorities for developing countries, Lancet 2007; 369:1314-7.

9. Boyle C, Alexander M. Public health research at the CDC: Implications for communication sciences and disorders. Journal of Communication Disorders 2005; 38:263-70.

10. Trenche MCB, Barzaghi L, Pupo AC. Cambio curricular: construcción de un nuevo proyecto pedagógico de formación en el área de fonoaudiología. Interface- Comunic. Saúde Educ. 2008; 12(27):697-711.

11. BRASIL. Portaria GM/MS № 154, de 24 de janeiro de 2008. Cria os Núcleos de Apoio à Saúde da Família. Disponível em: http://200.137.177.147/ sistemas_de_informacao/doc_tec_leg/siab/ portaria-n-154-nasf.pdf. Consulta em: 06/06/2010.

12. Johnson A. Promoting the Value of Audiology and Speech-Language Pathology in health Care. The ASHA Leader. 2006;10(17):52-3.

13. Barros PML, Oliveira PN. Perfil dos pacientes atendidos no setor de fonoaudiologia de um serviço público de Recife - PE. Rev. CEFAC 2010; 12(1): 128-33.

14. Lanzetta BP, Frota S, Goldfeld M. Acompanhamento da adaptação de próteses auditivas em crianças surdas. Rev. CEFAC 2010;12(3): 360-70. 
15. Goulart BNG, Chiari BM. Avaliação clínica fonoaudiológica, integralidade e humanização: perspectivas gerais e contribuições para reflexão. Rev Soc Bras Fonoaudiol. 2007;12(4):335-40.

16. Swanepoel DW, Störbeck C, Friedland $P$. Early hearing detection and intervention in South Africa. International Journal of Pediatric Otorhinolaryngology. 2009;73:783-6.

17. Theunissen M, Swanepoel DW. Early hearing detection and intervention services in the public health sector of South Africa. Int. J. Audiol. 2008;47:S23-S9.

18. Vaughan R. Evaluation and public health. Am J Publ Health 2004;94(3):360.

19. Malta DC, Leal MC, Costa MFL, Neto OLM. Inquéritos Nacionais de Saúde: experiência acumulada e proposta para o inquérito de saúde brasileiro. 2008; 11(Supl 1):159-67.

20. Chiari BM, Goulart BNG. The role of research methodology in the rational use of technology in monitoring and preventing communication disorders, An Acad Bras Cienc 2009; 81(3):497-502.

21. Minas Gerais a. Secretaria de Estado de Saúde. Plano Estadual de Saúde 2008-2011. Belo Horizonte: SES/MG, 2008. Disponível em: www. saude.mg.gov.br

22. Minas Gerais b. Secretaria de Estado de Saúde. Resolução SES no 1669 de 19 de novembro de 2008. Disponível em: http://www.saude.mg.gov.br/atos normativos/resolucoes/2008/RESOLUCaO\%20 SES\%20No 1669\%20DE\%2019\%20DE\%20 NOVEMBRO\%20DE\%202008.pdf

23. Mendes EV. As redes de atenção à saúde. Belo Horizonte: ESP/MG, 2009.

24. Kramers PGN. The ECHI project- Health indicators for the European Community. European Jounal of Public Health 2003; 13(3):101-6.

25. Bazzo LMF, Noronha CV. A ótica dos usuários sobre a oferta do atendimento fonoaudiológico no Sistema Único de Saúde (SUS) em Salvador. Ciência \& Saúde Coletiva. 2009;14(Supl 1):1553-64. 26. Ministério da Saúde. DATA SUS. Caderno de Informações em Saúde do estado de Minas Gerais, versão fevereiro de 2009. Disponível em: www. datasus.gov.br Consulta em 10/05/2010

http://dx.doi.org/10.1590/S1516-18462011005000088

RECEBIDO EM: 29/07/2010

ACEITO EM: 09/11/2010

Endereço para correspondência:

Juliana Nunes Santos

Rua Coronel Pedro Jorge, 170/201, Bairro Prado

Belo Horizonte - Minas Gerais

CEP: 30410-350

E-mail: jununessantos@yahoo.com.br
27. Minas Gerais. Secretaria de Estado de Saúde de Minas Gerais. Tabela dos Procedimentos Ambulatoriais do SUS/MG por Nível de Atenção. 2007. Disponível em:http://www.saude. mg.gov.br/politicas_de_saude/plano-diretorde-regionalizacao-pdr-novo/Carteira\%20 AMBULATORIAL\%20-\%202007.pdf. Consulta em: 07/06/2010

28. Pinheiro R, Ferla A, Silva JAG. Integrality in the population's health care programs. Ciênc. saúde coletiva 2007; 12(2): 343-9.

29. Kawachi I, Subramanian SV, AlmeidaFilho N. A glossary for health inequalities. Journal of Epidemiology and Community Health 2002;56:647-52.

30. Fang P, Dong S, Xiao J, Liu C, Feng X, Wang Y. Regional inequality in health and its determinants: Evidence from China. Health Policy. 2010; 04:14-25 31. Heritage Z. Inequalities, social ties and health in France. Public Health. 2009;23:e29-e34.

32. Lessa FJD, Miranda GMD. Fonoaudiologia e Saúde Pública. In: Brito ATB de. (Org.). Livro de Fonoaudiologia. São José dos Campos: Pulso Editorial, 2005, 460p.

33. Bazzo LMF. Informação em Saúde: subsídios para caracterização da oferta de serviços fonoaudiológicos do Sistema Único de Saúde (SUS) em Salvador. R. Ci. méd. biol. 2007; 6 (2):214-8.

34. Buarque APFC, Campos LCS, Reis FKW, Guedes JBR, Lima TFP, Pereira GFC, Silva HJ. Caracterização da oferta de fonoaudiólogos segundo macrorregiões do Brasil. Rev Soc Bras Fonoaudiol. (Supl Especial) 2009; 1550. Disponível em: http://www.sbfa.org.br/portal/anais2009/anais_ select. php?op=PR\&cid $=1550 \&$ tid $=1$

35. Bazzo LMF. Privação da oferta de serviços fonoaudiológicos no Sistema Único de Saúde (SUS) e a reforma do Estado: a mediação do debate. R. Ci. méd. biol. 2007; 6(2):190-6.

36. Moreira ML. Sistema de Informação de Saúde: A Epidemiologia e a Gestão de Serviço. Saúde e Sociedade, 1995; 4(1/2):43-5.

37. Costa $\mathrm{CH}$. Por uma saúde mais ampla. J Cons Fed Fonoaudiol. 2003; 8:5-6. 